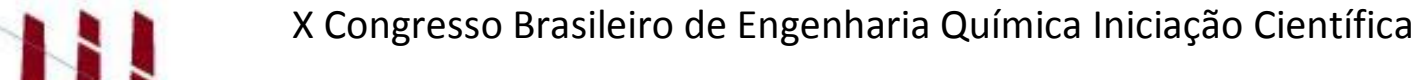 \\ "Influência da pesquisa em Engenharia Química no desenvolvimento tecnológico e industrial brasileiro" \\ Universidade Federal Rural do Rio de Janeiro Universidade Severino Sombra Vassouras -RJ-Brasil
}

\section{DESENVOLVIMENTO DE SOFTWARE PARA AUTOMATIZAÇÃO DO LABORATÓRIO PVT DE ÓLEOS PESADOS}

\author{
VOLPATTO*1, D. T.; SILVA², A. L.; SOUSA $^{3}$, A.O.; SOUZA ${ }^{4}$, D.F.S. \\ ${ }^{1}$ Aluno do DEQ/UFRN $\quad{ }^{2}$ Técnico do LabPVT/NUP-ER/UFRN $\quad{ }^{3}$ Professor do DFTE/UFRN \\ ${ }^{4}$ Professor do DEQ/UFRN \\ Departamento de Engenharia Química - Centro de Tecnologia - UFRN \\ Endereço-UFRN, Campus Universitário, Lagoa Nova, Natal, CEP. 59.072-970, RN, \\ email: domingos@eq.ufrn.br
}

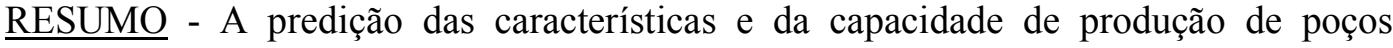
maduros de petróleo demanda experimentos do comportamento de fases do fluido nas condições de reservatório. Os experimentos são realizados em amostras de óleo (geralmente multifásicas), de composição desconhecida e obtida nas condições de pressão e temperatura do reservatório (acima de 6000 psi e $70{ }^{\circ} \mathrm{C}$ ). Inicialmente, determina-se a viscosidade e densidade das amostras para, em seguida, serem separadas nas frações líquidas e gasosas. Pequenas parcelas de cada uma das frações são direcionadas para: (a) caracterização por cromatografia; (b) separações multifásicas sequencial; (c) compressão e expansão sequencial; (d) determinação dos pontos de bolha e orvalho. Por fim, este conjunto extenso de resultados experimentais é reagrupado e utilizado na estimativa das condições do poço produtor. Essa laboriosa tarefa motivou o presente trabalho, que tem como objetivo a criação de software para automatização das análises PVT - Pressão, Volume e Temperatura - e geração de relatórios/resultados. O programa desenvolvido em linguagem Python possui interface amigável, gerador de gráficos, pacotes numéricos para estimativa de propriedades termodinâmicas e de equilíbrio de fases e comunicação com demais softwares para edição de relatórios (Word ${ }^{\circledR}$, Excel ${ }^{\circledR}$, Adobe ${ }^{\circledR}$ e outros). Para validação do software utilizou-se informações de dados experimentais disponibilizados na literatura.
\end{abstract}

Palavras chave: análise PVT, propriedades PVT, fluidos de reservatório de petróleo.

\section{INTRODUÇÃO}

Os ensaios experimentais PVT representam um conjunto de análises de comportamento de fases de óleos pesados em condições de reservatório. Estas análises são utilizadas na previsão da capacidade de produção e no estudo reológico do óleo
(Rutledge e Rajagopal, 2007) impactando na definição dos parâmetros de refino.

Os experimentos de análise PVT para óleos pesados têm inicio com a coleta de amostras em cilindros de amostragem. Neste caso, devem-se garantir as condições de pressão e temperatura do reservatório (Danesh, 1998). A etapa seguinte é a realização de uma

*Bolsista de Iniciação Científica FUNPEC/ANP/UFRN 
separação "flash" em condições ambiente e a determinação do volume superficial da fase gasosa e líquida. Após a análise, são realizadas amostragens das fases para determinação composicional do óleo por análises cromatográficas. $\mathrm{O}$ procedimento experimental seguinte é a determinação da pressão de saturação do óleo pesado (determinação do ponto de bolha) em uma célula de análise célula PVT. Neste equipamento torna-se possível variar o volume de amostra em relação à pressão. Os resultados obtidos representam uma curva de pressão versus volume (com a temperatura constante) onde o ponto de inflexão indica à pressão de bolha da mistura (Rutledge e Rajagopal, 2007).

A emissão do parecer final sobre as características do reservatório perpassa todos os experimentos citados acima, bem como o agrupamento ordenado do conjunto de resultados obtidos. Sendo assim, o presente trabalho tem como objetivo principal viabilizar a coleta e tratamento do conjunto de resultados obtidos com os experimentos de análise PVT concentrando todas as informações experimentais em um único banco de dados.

\section{MATERIAL E MÉTODOS}

O trabalho é composto, essencialmente, por uso de ferramentas computacionais e a sistematização dos procedimentos realizados em um laboratório de análises PVT, onde:

a) Para a elaboração do programa de forma geral, foi utilizada a linguagem Python 2.7 (Lutz, 2009) e recursos de sua biblioteca padrão.

b) Para a construção da interface gráfica, utilizou-se a biblioteca open source wxPython 2.9 (Rappin e Dunn, 2006) em conjunto com o construtor wxGlade v0.6.7 (Grohmann, 2013).

c) Demais recursos foram inseridos a partir de pacotes científicos como o SciPy (Scipy Community, 2013) e a biblioteca Pint (Grecco, 2013), que é voltada para utilização de sistemas de unidades e conversões. d) Para edição de código e junção das bibliotecas, foi usado o Portable Python 2.7.5.1.

Todas as ferramentas utilizadas têm como característica a licença livre, o que garante o uso legal delas.

Dentre os métodos padrões de análises PVT associados ao óleo pesado, o presente trabalho aborda os dados do Constant Composition Expansion (CCE) - expansão à composição constante -, que tem como finalidades determinar a pressão do ponto de bolha, a densidade do óleo subsaturado, compressibilidade isotérmica do óleo e o comportamento volumétrico bifásico em pressões inferiores ao ponto de bolha (Whitson e Brulé, 2000), tudo à temperatura constante. Os dados de pressão e volume utilizados neste trabalho são oriundos de um experimento do Laboratório PVT do NUP-ER (UFRN) - que foram realizados uma amostra de poço obtido em campo, especificamente, Xaréu - e outros dados encontrados na literatura (Whitson e Brulé, 2000) que foram também inseridos no programa via interface gráfica. No caso dos dados obtidos por meio de experimentos, realizou-se uma importação de dados de um arquivo com extensão csv (comma separated values), que foi gerado pela maquina conectada à célula PVT (Chandler Engineering modelo 3000-L). Esses dados foram adquiridos através de um sistema de aquisição PC - célula PVT, fornecido pelo fabricante. Após inserção ou importação de dados, tornase possível a confecção de gráficos de pressão versus volume, que viabilizam o estudo do comportamento da amostra.

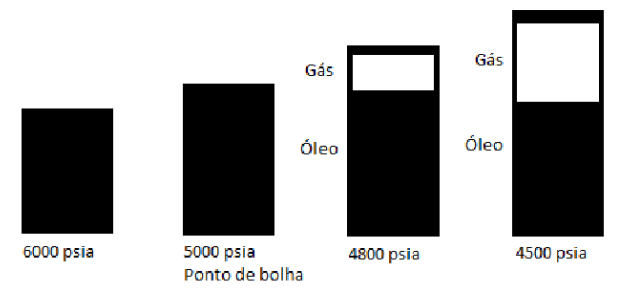

Figura 1 - Esquema do experimento CCE.

Seguindo às necessidades de armazenamento de dados apropriados, também é concedida a entrada de informações referente às condições da amostra e às características da 
formação e do poço. Pode-se, também, inserir a composição da amostra, posteriormente à análise cromatográfica, tanto em porcentagem molar como em mássica, ficando a critério do usuário. Com esse conjunto de dados em mãos, o programa oferece a funcionalidade de
A figura 3 mostra um diagrama explicativo sobre a organização do programa até o momento da escrita deste texto. Também é possível abrir o editor de relatórios, que se encontra no menu "Editar" e gerar relatórios no menu "Arquivo", no item "Gerar relatório".

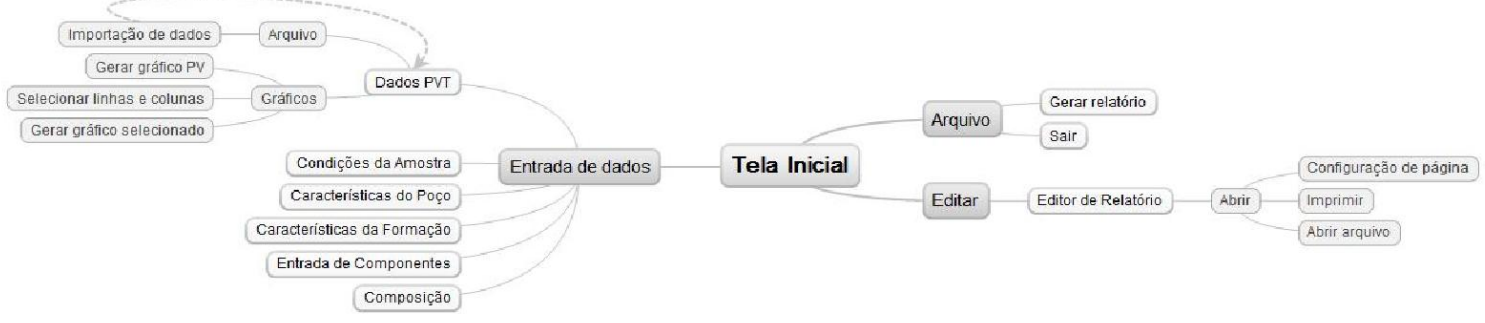

Figura 3 - Diagrama do aPVT.

gerar um relatório padrão ANSI, assim como a possibilidade de impressão e edição através de um editor interno ao software.

\section{RESULTADOS E DISCUSSÕES}

Convém destacar que o produto do trabalho é um software, então os resultados e as discussões apresentadas estão direcionadas ao uso da ferramenta.

Ao abrir o programa, inicia-se a tela principal do "aPVT", mostrada na figura 2 , de onde é possível acessar as opções de entrada de dados, que possui os campos para escolha de sistemas de unidades (em fase de implementação), para entrada dos componentes presentes em uma amostra, entrada da composição de uma amostra, entrada de dados PVT em uma tabela, entrada de dados das condições da amostra e características da formação e do poço. A tela inicial possui o seguinte aspecto:

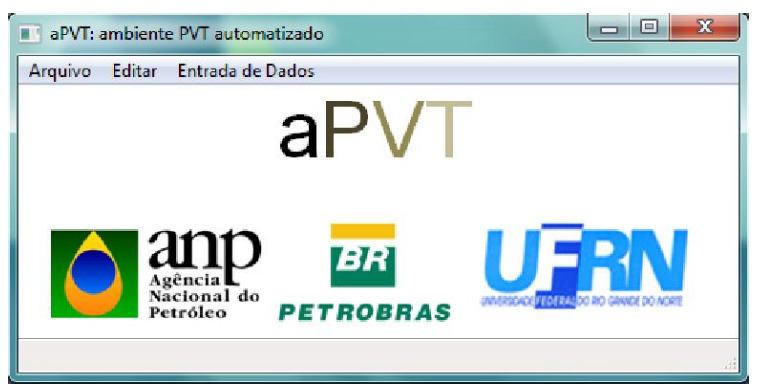

Figura 2 - Tela inicial do aPVT.
No que se refere ao experimento CCE, é de interesse o campo "dados PVT" no menu "Entrada de Dados". Ao selecionar esse campo, o programa leva o usuário a uma janela que possui uma tabela (figura $4 a$ ), na qual o usuário pode entrar com dados PVT numa organização que lhe convir, ou pode importar uma tabela que possua extensão $c s v$ (figura 4b). Em ambos os casos, o usuário tem a opção de gerar gráficos. Caso a importação seja oriunda da célula PVT do Laboratório PVT do NUP-ER (UFRN), no menu "Gráficos" existe a opção "Gerar gráfico PV", que já é configurado para confeccionar gráficos no padrão dos dados gerados na célula. Senão, o usuário pode escolher o intervalo de linhas e as colunas que deseja plotar, inclusive pode nomear os eixos do gráfico.

Para realizar tal feito, basta selecionar as regiões do gráfico em "Selecionar linhas e colunas", presente em "Gráficos". Isso abrirá uma janela aonde será feita todas as definições ditas anteriormente. Depois, basta clicar em "Gerar gráfico selecionado". Abaixo, encontra-se a imagem das janelas previamente anunciadas: 


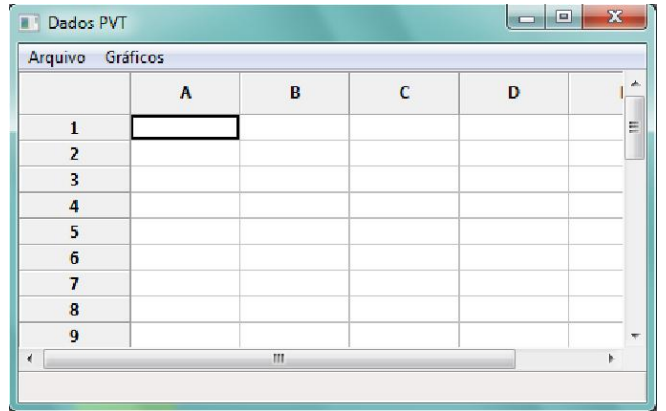

(a)

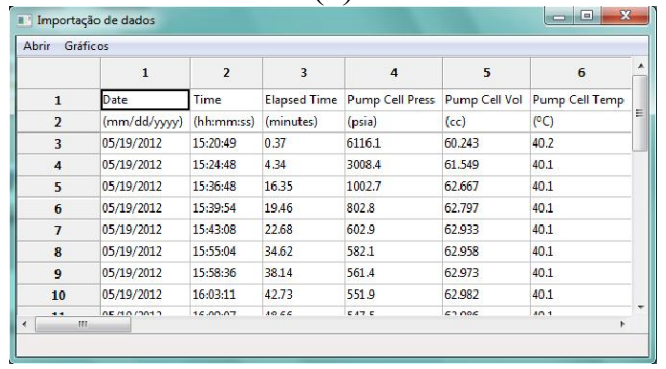

(b)

Figura 4 - (a) Telas dos dados PVT sem importação; (b) Tela com dados PVT importados.

Utilizando-se de dados encontrados na literatura (Whitson e Brulé, 2000) e os obtidos por meio de experimento CCE no Laboratório PVT (via importação no aPVT), obteve-se os gráficos de pressão vs volume, mostrados abaixo pelas figuras $5 \mathrm{a}$ e $5 \mathrm{~b}$.

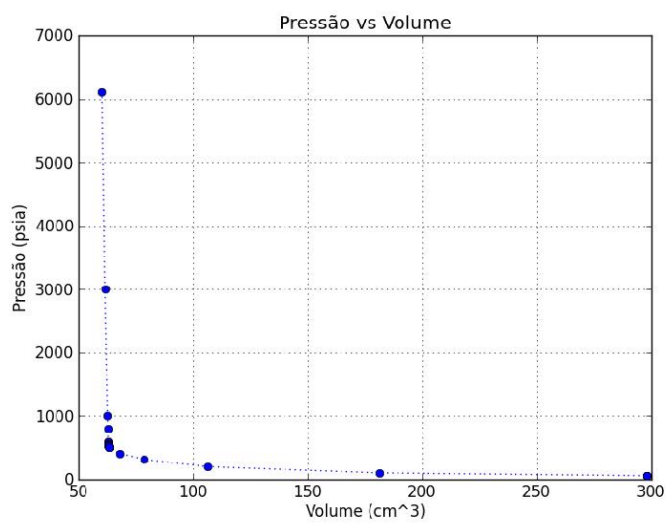

(a)

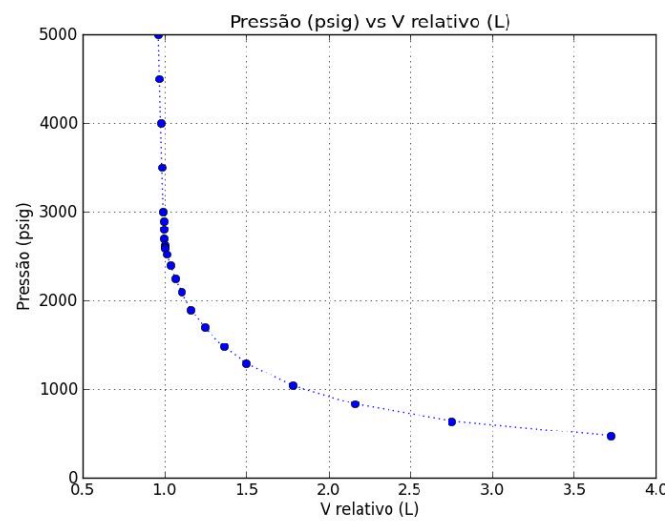

(b)

Figura 5 - (a) Gráfico Pressão vs Volume do Laboratório PVT; (b) Gráfico Pressão vs Volume com dados de Whitson e Brulé (2000).

No segmento da elaboração de relatórios, entram as janelas de entrada de dados das Condições da Amostra (figura 6c), das Características do Poço (figura 6b) e da Formação (figura 6a), assim como a Composição. Estas informações correspondem a ficha de entrada da amostra no laboratório e são obrigatoriamente preenchidas por um técnico.

\begin{tabular}{|c|c|c|}
\hline IT Entre com os dados da Formacao & \multicolumn{2}{|c|}{\begin{tabular}{|l|l|l|}
$\square$ & 回 & $x$ \\
\end{tabular}} \\
\hline \multicolumn{2}{|l|}{ Formacao } & - \\
\hline \multicolumn{3}{|l|}{ Nome } \\
\hline \multicolumn{3}{|l|}{ Primeiro Poco Completado } \\
\hline \multicolumn{3}{|l|}{ Pressao de Reservatorio Original } \\
\hline \multicolumn{3}{|l|}{ RGO Produzido Originalmente } \\
\hline \multicolumn{3}{|l|}{ Taxa de producao, B/D } \\
\hline \multicolumn{3}{|l|}{ Temperatura do separador } \\
\hline \multicolumn{3}{|l|}{ Pressao do separador } \\
\hline \multicolumn{3}{|l|}{ Densidade do oleo } \\
\hline \multicolumn{3}{|l|}{ Datum } \\
\hline \multicolumn{3}{|l|}{ Gas cap original } \\
\hline ब & $\longrightarrow$ & \\
\hline
\end{tabular}

(a) 


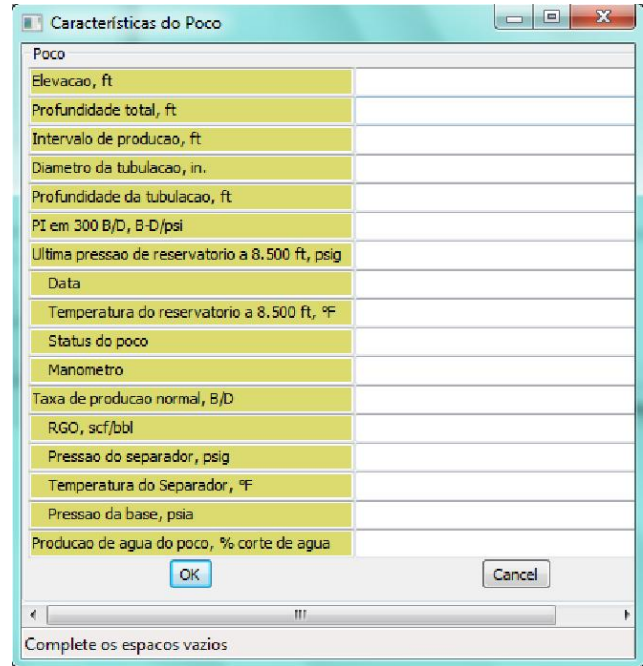

(b)

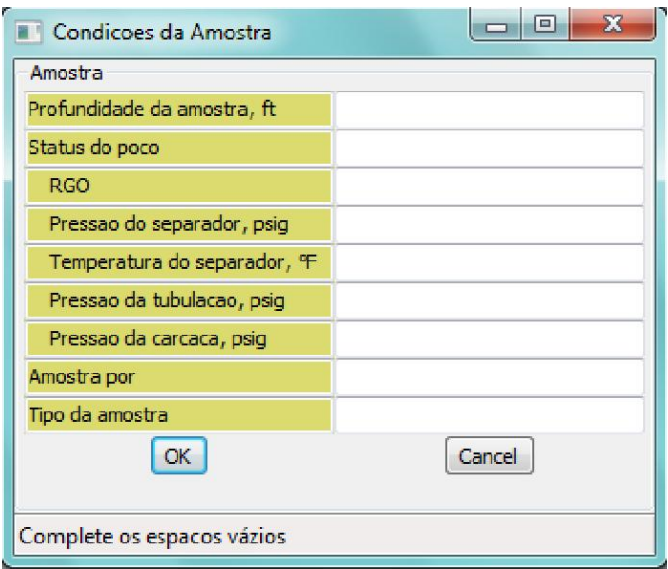

(c)

Figura 6 - Janelas dos dados (a) Formação;

(b) Poço; (c) Amostra.

Após o ensaio de separação "flash" nas condições ambiente - flash zero, duas amostras são obtidas (fase líquida e fase gasosa). As amostras são enviadas ao laboratório de análises cromatográficas para realizar a determinação da composição das respectivas fases. Esse processo é de suma importância para a realização dos demais cálculos em PVT.

As janelas apresentadas nas figuras 7(a) e 7(b) representam a seqüência de informações necessárias para o preenchimento do fichário relacionado a cromatografia.

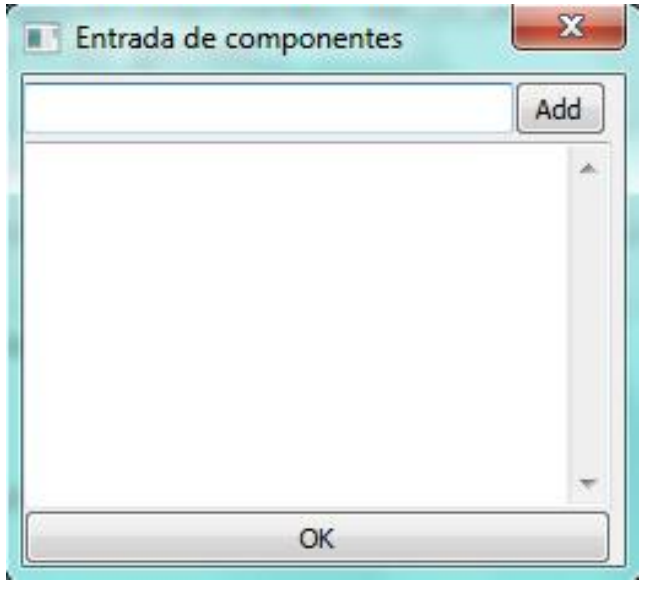

(a)

\begin{tabular}{|c|c|c|}
\hline \multicolumn{2}{|c|}{ III Composição } & \begin{tabular}{l|l|l|} 
& 回 & $\mathrm{X}$ \\
\end{tabular} \\
\hline \multicolumn{3}{|l|}{ Arquivo } \\
\hline & $\%$ mol & $\% w t$ \\
\hline $\mathrm{H} 2 \mathrm{~S}$ & 0 & 0 \\
\hline $\mathrm{CO} 2$ & 0,91 & 0,43 \\
\hline N2 & 0,16 & 0,05 \\
\hline Methane & 36,47 & 6,24 \\
\hline Ethane & 9,67 & 3,10 \\
\hline Propane & 6,95 & 3,27 \\
\hline i-butane & 1,44 & 0,89 \\
\hline n-butane & 3,93 & 2,44 \\
\hline i-pentane & 1,44 & 1,11 \\
\hline n-pentane & 1,41 & 1,09 \\
\hline Hexanes & 4,33 & 3,97 \\
\hline $\mathrm{C7}+$ & 33,29 & 77,41 \\
\hline \%total & 100.0 & 100.0 \\
\hline
\end{tabular}

(b)

Figura 7 - Janelas dos dados de composição (a) Entrada dos componentes; (b) Composição.

Com o preenchimento dos dados das janelas acima, é possível então, através do menu "Arquivo", gerar o relatório. Caso haja a necessidade de editar o relatório ou mesmo imprimi-lo, o editor de relatório realiza isso. No relatório gerado, devem constar os dados armazenados nas janelas da Figura 6 e da Figura 7 (análise composição). 


\section{CONCLUSÃO}

Ainda no estágio inicial, o software aPVT apresenta-se como uma ferramenta computacional versátil para a geração de relatórios e importação dados. Estas funcionalidades estão aliadas à flexibilidade de confecção de gráficos experimentais e análise de dados oriundos de experimentos PVT além de permitir padronizar todos os procedimentos laboratoriais em um único ambiente/software.

\section{REFERÊNCIAS}

DANESH, A. (1998), PVT and Phase Behavior of Petroleum Reservoir Fluids. Editora Elsevier, Amsterdam.

GRECCO, H. E. Pint Documentation. Disponível em: $<$ https://media.readthedocs.org/pdf/pint/lat est/pint.pdf $>$. Acesso em: 24 set. 2013.

GROHMANN, C. WxGlade manual. Disponível em: $<$ http://wxglade.sourceforge.net/manual $/ \mathrm{m}$ anual.pdf $>$. Acesso em: 24 set. 2013.

LUTZ, M. (2009), Python: Pocket Reference. Editora O'reilly, Sebastopol.

RAPPIN, N., DUNN, R. (2006), WxPython In Action. Editora Manning, Greenwich.

RUTLEDGE, L. A. M., RAJAGOPAL, K. (2007), "Determinação de Ponto de Bolha de um Óleo Vivo a partir de Dados PVT" $4^{\circ}$ Pdpetro, Campinas-Sp, p. 1-7.

SCIPY COMMUNITY. SciPy Reference Guide. Disponível em: $<$ http://docs.scipy.org/doc/scipy/scipyref.pdf $>$. Acesso em: 24 set. 2013.

WHITSON, C. H., BRULÉ, M. R. (2000), Phase Behavior. Editora Society Of Petroleum Engineers, Internacional. (SPE Monograph Series).

\section{AGRADECIMENTOS}

À Agência Nacional do Petróleo, Gás Natural e Biocombustíveis, à Petrobras e à FINEP pelo fomento para a realização do presente trabalho. 